\title{
Computing Phrasal-signs in HPSG prior to Parsing
}

\author{
Kentaro Torisawa and Jun'ichi Tsujii \\ Department of Information Science, University of Tokyo, \\ Hongo 7-3-1, Bunkyo-ku, Tokyo, 113, Japan \\ \{torisawa,tsujii\}ois.s.u-tokyo.ac.jp
}

\begin{abstract}
This paper describes techniques to com pile lexical entries in IIPSG (Pollard and Sag, 1987; Pollard and Sag, 1993)-style grammar into a sot of finito state antomati. The states in antomata are possible signs derived from lexical entries and contain information raised from the lexical entries. The automata are augmented with feature structures used by a partial unification routine and delayed/frozen definite clause programs.
\end{abstract}

\section{Introduction}

Onr ain is to build an efficiont and robust HPSGbased parser. IIPSG has been regarded as a sophisticated but fragile and inofficiont framowork. However, its principle-based architecture cnables a parser to handle real world texts only by giving concise core grammar, including principles and templates for lexical entries, default lexical entries(Horiguchi et al., 1995). 'The architecture is different from those of conventional unificationbased formalisms which require hundreds of $\mathrm{Cl} G$ skeletons to parse real world texts.

However, these design principles of IIPSG have draw-backs in parsing cost. That is, signs/foature structures corresponding to non-terminal symbols in CF $\mathrm{X}$ becone visible only after applying principles and a parser has to create feature structures one by one using unification. In addition, identity checking of non-terminal symbols used to eliminate spurious signs must be replaced with subsumption checking, which further deteriorates efficiency.

Our grammar compiler computes skeletal part of possible phrasal-signs fron individual loxical cutries prior to parsing, and gencrates a sot of finite state antomata from lexical entries to avoid the above draw-backs. We call this operation Offline raising and an antomaton thes generated is called a Lexical Entry Automaton (IA). Its states corresponds to part of signs and each transition between states corresponds to application of a rule schema, which is a non-lexical component of grammar.

Our parsing algorithm adopts a two-phased parsing method.

Phase 1 Bottom-np chart-like parsing with LAs.

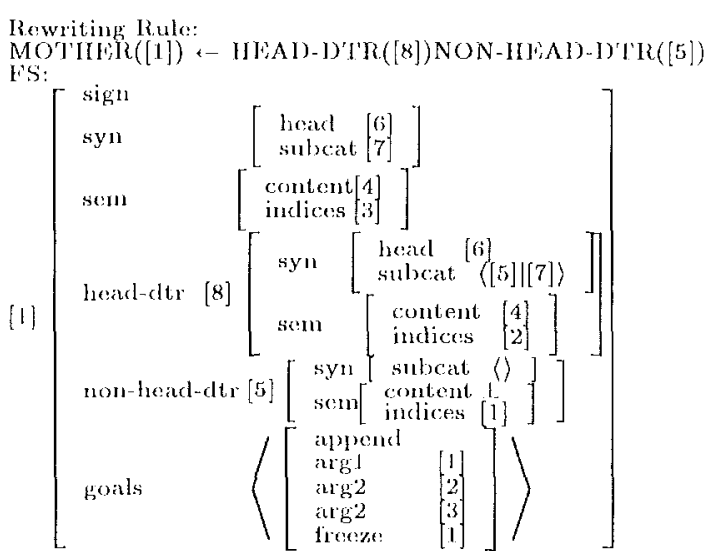

Figure 1: $\Lambda$ is example of a rule schema.

Phase 2 Computing part of feature structures which camnot be computed at compile-time.

We call the foature structures that are reprosented as states in automata and are computed at compile-time Core-structures, and the feature structures which are to be computed in Phase 2 , Sub-structures. In Phase 1 parsing, a coresitructure correspond to a state in an $]$.A. The cost of computing sub-structures at Phase 2 is mininized by Dependency Analysis and Partial Unification.

Tho next soction describes rule schemata, central components of the formalism, and gives a definition of Definite Clatuse Programs. Section 3 describes how to obtain $L \Lambda$ s from lexical entries and kow to perform the Phase 1 parsing. Section $4 \mathrm{cx}-$ plains the Phase 2 Parsing algorithm. A parsing example is presented in Section 5. The offectiveness of our method is exemplified with a serios of experiments in Section 6 .

\section{Rule Schomata and Definite Clause Programs}

Our formalism has only one type of component as non-lexical components of grammar, i.e., rule schemata. ${ }^{1}$ An cxample is shown in Figure 1. A rule schema consists of the following two itoms.

\footnotetext{
IIn our current system, rule schemata are generated from principles and rewriting rules according to a specification given by a programmer.
} 
rule(R) a rewriting rule without specific syntactic categories;

$\mathbf{f s}(\mathbf{R})$ a feature structure.

A characteristic of HPSG is in the flexibility of principles which demands complex operations, such as append or subtraction of list-value feature structures. In our formalism, those operations are treated by a Definite Clause Program. A DCP can be seen as a logic program language whose arguments are feature structures. An auxiliary term, a query to a DCP augmenting a rule schema, is embedded in a feature structure of a rule schema as the value of goals. The rule schema in the example has an auxiliary term, append $([1],[2],[3])$.

The bottom-up application of the rule schema $R$ is carried out as follows. First, two daughter signs are substituted to the HEAD-DTR position and NON-HEAD-DTR position of the rewriting rule rule $(R)$. Then, the signs are unified with the head-dtr value and the non-head-dtr value of the feature structure of the schema, $f s(R)$. Finally, the auxiliary term for DCPs given in the schema is evaluated.

Our definition of a DCP has a more operational flavor than that given by Carpenter(Carpenter, 1992). The definition is crucial to capture the correctness of our method. ${ }^{2}$

Definition 1 (DCP) $A$ definite clause program (DCP) is a finite set of feature structures, each of which has the following form.

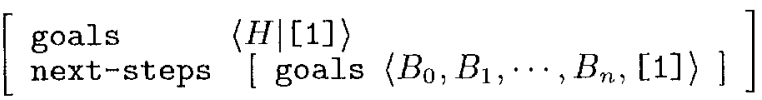

${ }^{3}$ where $0 \leq n$ and $H, B_{0}, \cdots, B_{n}$ are feature structures.

A feature structure of the above form corresponds to a clause in Prolog. $H, B_{0}, \cdots, B_{n}$ corresponds to literals in Prolog. $H$ is the head and $B_{0}, \cdots, B_{n}$ are literals in the body of a clause.

Definition 2 (Execution of DCP) Execution of a DCP $P$ for the query,

$$
\text { Query }=\left[\text { goals }\left\langle q_{0}, q_{1}, \cdots q_{l}\right\rangle\right]
$$

is a sequence of unification,

$$
\text { Query } \sqcup r_{1} \sqcup r_{2} \sqcup \cdots \sqcup r_{n}
$$

where $r_{i}=\left[\begin{array}{ll}\text { (next-steps) } & C_{i}-1\end{array}\right], C_{i} \in P$ or $C_{i}=[$ goals \langle\rangle$]$. If the execution is terminated, $C_{n}$ mist be unifiable with [ goals \langle\rangle$]$. In this case, we call the sequence $\left\langle r_{1}, \cdots, r_{n}\right\rangle$ a resolution sequence.

${ }^{2}$ Though, through the rest of the paper, we treat the definition as if it were used in an actual implementation, the actual implementation uses a more efficient method whose output is equivalent with the result obtained by the definition.

$$
\begin{aligned}
& { }^{3}\left\langle B_{0}, \cdots, B_{n},[1]\right\rangle \text { is an abbreviation of } \\
& {\left[\begin{array}{l}
\text { first } B_{0} \\
\text { rest }
\end{array} \cdots\left[\begin{array}{ll}
\text { first } & B_{n} \\
\text { rest } & {[1]}
\end{array}\right]\right]}
\end{aligned}
$$

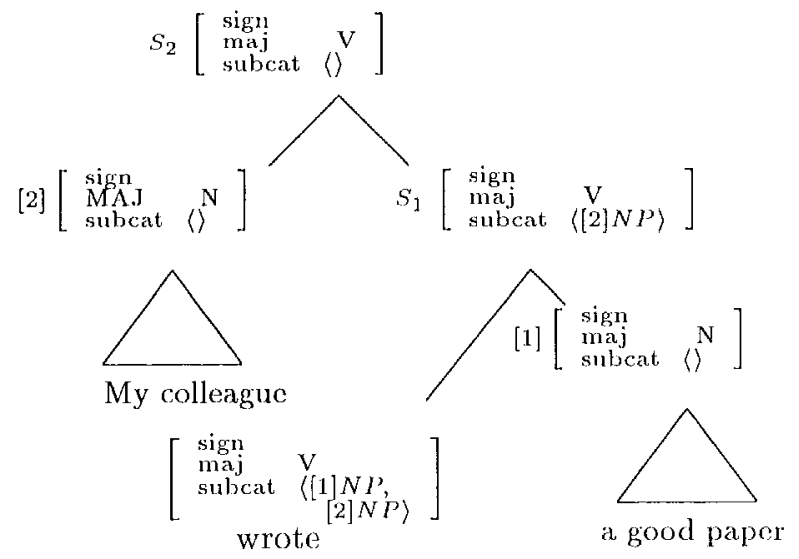

Figure 3: A parsing cxample

(next-steps) ${ }^{i-1}$ Igoals of Query $\sqcup r_{1} \cup r_{2} \sqcup \cdots \sqcup$ $r_{i}$ represents the goals which are to be solved in the steps following the $i$-th step. The goals are instantiated by the steps from the first one to $i$-th one, through structure sharings. The result of cxecution in a Prolog-like sense appears in the query. Figure 2 is an example of execution for the query append ( $[\mathrm{a}],[\mathrm{b}], \mathrm{X})$, whose definition is based on a standard definition of append in Prolog.

Given this defmition of DCPS, an application of a rule schema to two daughter signs $D_{1}$ and $\mathrm{D}_{2}$ can be expressed in the following form, where $\left\langle r_{1}, r_{2}, \cdots, r_{n}\right\rangle$ is a resolution sequence:

$M=\left[\begin{array}{ll}\text { head-dtr } & D_{1} \\ \text { non-head-dtr } & D_{2}\end{array}\right] \sqcup f s(R) \sqcup r_{1} \sqcup r_{2} \sqcup \cdots \sqcup r_{n}$

\section{Lexical Entry Automata}

This section presents a Lexical Entry Automaton (LA). The inefficiency of parsing in HPSG is due to the fact that what kind of constituents phrasal-signs would become is invisible until the whole sequence of applications of rule schemata is completed. Consider the parse tree in Figure 3. The phrasal-signs $S_{1}$ and $S_{2}$ are invisible until a parser creates the feature structures describing them, using expensive unification.

Our parsing method avoids this on-line construction of phrasal-signs by computing skeletal part of parse trees prior to parsing. In Figure 3 , our compiler gencrates $S_{1}$ and $S_{2}$ only from the lexical entry "wrote," without specifying the non-head danghters indicated by the triangles in Figure 3. Since the non-head daughters are tokenidentical with subcat values of the lexical entry for "wrote", the obtained skelctal parse tree contains the information that $S_{1}$ takes a noun phrase as object and $S_{2}$ selects another nomn-phrase. Then unifying those non-head daughters with actual signs constructed from input, parsing can be done. An LA expresses a set of such skeletal parse trees. A state in an LA corresponds to a phrasalsign such as $S_{1}$ and $S_{2}$. They are called corestructures. A transition arc is a domination link between a phrasal-sign and its head daughter, and its condition for transition on input is a non-head 


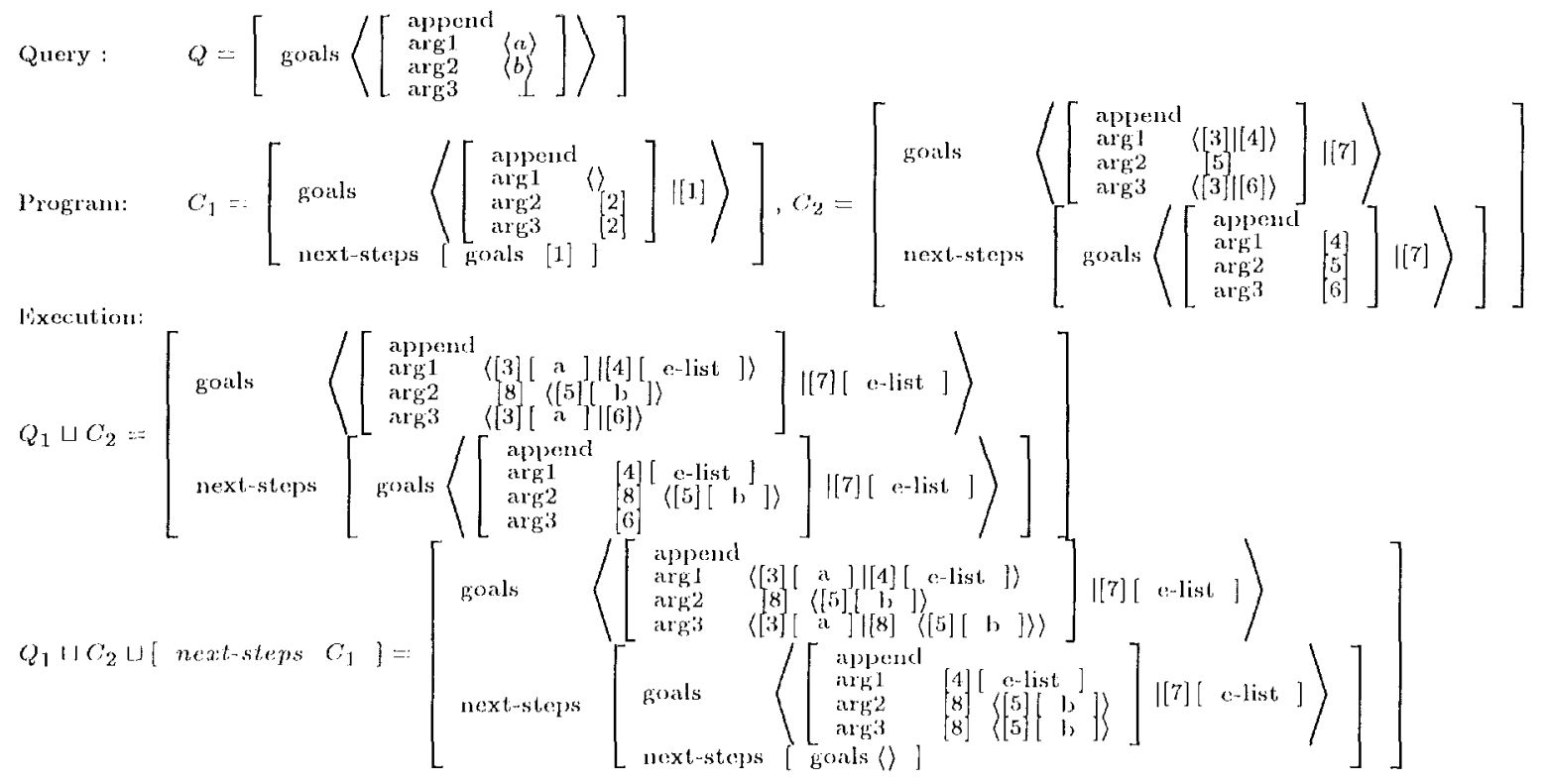

Figure 2: An oxample of DCP's execution

daughter, such as signs tagged [1] and [2] in Fig ure 3. Kasper et al. presented an idea similar to this off-line raising in their work on JIPSG-'TAG compiler(Kasper et al., 1995). The difference is that our algorithm is based on substitution, not adjoining. Furthermore, it is not clear in their work how off-line raising is used to improve efficiency of parsing.

Before giving the definition of IASs, we define the notion of a quasi-sign, which is part of a sign and constitutes I.As.

Definition 3 (quasi-sign(n)) For a given integer $n$, a feature structure $S$ is a quasi-sign(n) if it has some of the following four attributes: syn, sem, head-dtr, non-head-dtr and does not have values for the paths (head-dtr + non-head-dtr $)^{n}$.

A quasi-sign. $n$ ) cannot represent a parse treo whose height is more than $n$, while a sign can express a parse tree with any height. Through the rest of this paper, we often extract a quasi-sign $(n)$ $S$ from a sign or a quasi-sign $\left(n^{\prime}\right) S^{\prime}$ where $n \leq$ $n^{\prime}$. This operation is denoted by $S=\operatorname{ex}\left(S^{\prime \prime}, n\right)$. This moans that $S$ is equivalent to $S^{\prime}$ except for the attributes head-dtr and non-head-dtr whose root is the (head-dtr + non-head-dtr) ${ }^{n}$ value in $S^{\prime}$. Note that $S$ and $S^{\prime}$ are completely different entities. In other words, $S$ and $S^{\prime}$ pose different scopes on structure sharing tags. In addition, we also extract a feature structure $F$ reached by a path or an attribute $p$ in a feature structure $F^{\prime \prime}$. Wo denote this by $F=\operatorname{val}\left(F^{\prime}, p\right)$ and regard $F$ and $F^{\prime \prime}$ as different entities.

\section{Definition 4 (Lexical Entry Automaton(LA))} A Lexical Entry Automaton is a tuple $\left\langle Q, A, \varphi_{0}\right\rangle$ where,

$Q$ : a set of states, where a state is a quasi-sign $(0)$.
$A$ : a set of trunsition ares between states, where a transition arc is a tuple $\left\langle q_{d}, q_{m}, N, D, R\right\rangle$ where $q_{d}, q_{m} \in Q, N$ is a quasi-sign $(0), D$ is a quasi-sign (1) and $R$ is a rule schema.

$q_{0}$ : the initial state, which corresponds to a lexical entry.

In a transition are $\left\langle q_{d}, q_{m}, N, D, R\right\rangle, q_{m}$ denotes the destination of the transition are, and $q_{d}$ is the root of the arc. The $N$ is a non-head daughter of a phrasal-sign, i.e., the destination state of the transition, and expresses the input conclition for the transition. The $D$ is used to ropresent the dependency between tho mother sign and the danghters through structure sharings. This is called a Dependency Feature Structure(DFS) of the transition arc, the role of which will be discussed in Section 4. $R$ is the rule schema used to create this arc.

An $L \Lambda$ is generated from a lexical entry $l$ by the following recursive procedure:

1. Let $\mathcal{S}$ be $\{l\}, \mathcal{A}$ bo an empty set and $s_{d}=l$

2. For oach rule schema $R$, and for cach of its each resolution sequence $\left\langle r_{1}, \cdots, r_{n}\right\rangle$ obtain,

$$
\begin{aligned}
D= & {\left[\text { head-dtr } s_{d}\right] } \\
& \sqcup f s(R) \sqcup r_{1} \sqcup \cdots \sqcup r_{n}
\end{aligned}
$$

and if D is a fcature structure, obtain $s_{m}=\operatorname{ex}(D, 0)$ and $N=\operatorname{cx}(\operatorname{val}(D$, non-head-dtr $), 0)$.

3 . If $\mathrm{D}$ is a feature structure,

- If there is a state $s_{m}^{\prime} \in \mathcal{S}$ such that $s_{m}^{\prime} \approx$ $s_{m},{ }^{4}$ let $s_{m}$ be $s_{m}^{\prime}$. Otherwise, add $s_{m}$ to $\mathcal{S}$.

- If there is no $T r=\left\langle s_{d}^{\prime \prime}, s_{m}^{\prime \prime}, N^{\prime \prime}, D^{\prime \prime}, R\right\rangle \in$ $\mathcal{A}$ such that $s_{m} \approx s_{m}^{\prime \prime}, s_{d} \approx s_{l}^{\prime \prime}, N \approx$

${ }^{4}$ For any foalure structures $f$ and $f^{\prime}, f \approx f^{\prime}$ ifl $f \sqsubseteq f^{\prime}$ and $f^{\prime} \sqsubseteq f$ 


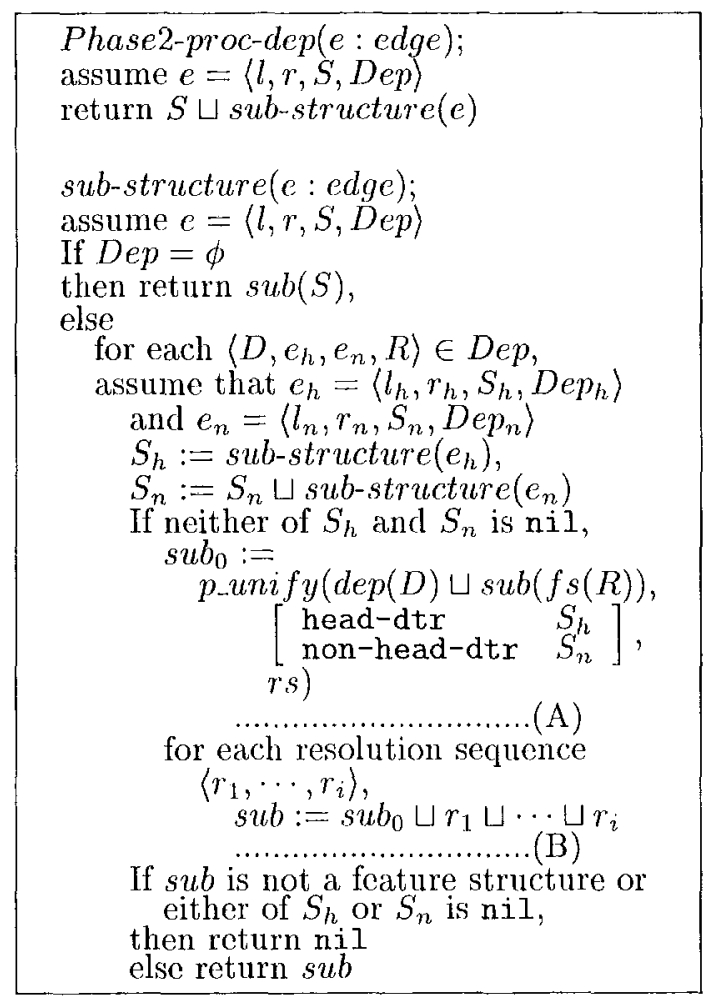

Figure 4: A recursive procedure for the Phase 2

$$
\begin{aligned}
& N^{\prime \prime} \text { and } D \approx D^{\prime \prime} \text {, then, add the tuple } \\
& \left\langle s_{d}, s_{m}, N, D, R\right\rangle \text { to } \mathcal{A} \text {. }
\end{aligned}
$$

4. If the new quasi-sign $(0)\left(s_{m}\right)$ was added to $\mathrm{S}$ in the previous step, let $s_{d}$ be $s_{m}$ and go to Step 2.

When this terminates, $\langle\mathcal{S}, \mathcal{A}, l\rangle$ is the LA for $l$. The major difference of Step 2 and the normal application of a rule schema is that non-head-dtr values are not specified in Step 2. In spite of this underspecification, certain parts of the non-head-dtr are instantiated because they are token-identical with certain values of the head-dtr domain. By unifying non-head-dtr values with actual signs to be constructed from input sentences, a parser can obtain parsing results. For more intuitive explanation, see (Torisawa and Tsujii, 1996).

However, this simple LA generation algorithm has a termination problem. There are two potential causes of non-termination. The first is the generative capacity of a feature structure of a rule schema, i.c., a rule schema can generate infinite variety of signs. The second is non-termination of the execution of DCP in Step 2 because of lack of concrete non-head daughters.

For the first case, consider a rule schema with the following feature structure.

$$
\left[\begin{array}{l}
\text { syn } \\
\text { head-dtr }
\end{array}\left[\begin{array}{l}
\text { counter }\langle b a r \mid[1]\rangle] \\
\text { syn [ counter [1] ] ] }
\end{array}\right]\right.
$$

Then, this can generate an infinite sequence of signs, each of which contains a part, [ counter $\langle b a r, b a r, \cdots, b a r\rangle]$ and is not equivalent to any previously generated sign. In order to resolve this difficulty, we apply the restriction (Shieber, 1985) to a rule schemata and a lexical entry, and split the feature structure $F=f s(R)$ of a rule schema $R$ or a lexical entry $F=l$ into two, namely, core $(F)$ and $\operatorname{sub}(F)$ such that $F=\operatorname{core}(F) \sqcup \operatorname{sub}(F)$. The definition of the restriction here is given as follows.

Definition 5 (paths) For any node $n$ in a feature structure $F$, paths $(n, F)$ is a set of all the paths that reaches $n$ from the root of $F$.

Definition 6 (Restriction Schema) $A$

restriction schema $r s$ is a set of paths.

Definition 7 (Res) $F^{\prime \prime}=\operatorname{Res}(F, r s)$ is a maximal feature structure such that each node $n$ in $F^{\prime}$ satisfies the following conditions.

- There is a node $n_{o}$ in $F$ such that $\operatorname{paths}\left(n_{o}, F^{\prime}\right)=\operatorname{paths}\left(n, F^{\prime}\right)$ and $\operatorname{type}(n)=$ type $\left(n_{o}\right)$.

- For any $p \in \operatorname{paths}\left(n, F^{\prime}\right)$, there is no path $p_{r} \in r s$ which prefixes $p$.

Res eliminates the feature structure nodes which is specified by a restriction schema. For a certain given restriction schemars, core $(f s(R))=$ $\operatorname{Res}(f s(R), r s)$ and $s u b(f s(R))$ is a minimal feature structure such that core $(f s(R)) \cup$ $s u b(f s(R))=f s(R)$. The nodes eliminated by Res must appear in $s u b(f s(R))$. In the example, if we add (syn, counter) to a restriction schema and replace $f s(R)$ with core $(f s(R))$ in the algorithm for generating $L A s$, the termination problom does not occur because LAs can contain a loop and equivalent signs are reduced to one state in LAs. The $s u b(f s(R))$ contains the syn/counter, and the value is treated at Phase 2.

The other problem, i.e., termmation of DCPs, often occurs because of underspecification of the non-head-dtr yalues. Consider the rule schema in Figure 1. The append does not terminate at Phase 2 because the indices value of non-head daughters is $[\perp]$. (Consider the case of executing append $(X,\langle b\rangle, Y)$ in Prolog. We introduce the freeze functor in Prolog which delays the evaluation of the second argument of the functors if the first argument is not instantiated. For instance, freeze $(X, \operatorname{append}(X,[b], Z))$ means to delay the evaluation of append until $X$ is instantiated. We introduce the functor in the following form.

$$
\left[\text { goals }\left\langle\left[\begin{array}{cc}
\text { append } \\
\text { arg1 } & {[1]} \\
\text { arg2 } & \langle 3\} \\
\text { arg3 } & {[2\}} \\
\text { freeze } & {[1]}
\end{array}\right]\right\rangle\right]
$$

This means the resolution of this query is not performed if [1] is [ 1 ]. The delayed evaluation is considered later when the non-head-dtr values are instantiated by an actual sign. Note that this change does not affect the discussion on the correctness of our parsing method, becanse the difference can be secn as only changes of order of unification.

Now, the two phases of our parsing algorithm can be described in more detail.

Phase 1 : Enumerate possible parses or edges in a chart only with unifiability checking in a bottom-up chart-parsing like manner. 
Phase 2: For completed parse trees, compute sub-structures by DFSs, sub(fs(R)) for each schema $R$ and frozen $\mathrm{DCl}$ programs.

Note that, in Phase 1, unification is replaced with unifiability checking, which is more efficient than unification in terms of space and time. The intended sirle effect by unification, such as building up logical forms in sem values, is computed at Phase 2 only for the parse trees covering the whole input.

\subsection{Phase 1 Parsing}

The Phase 1 parsing algorithm is quite similar to a bottom-up chart parsing for $\mathrm{CFG}$. The algorithm has a chart and odges.

Definition 8 (edge) An edge is a tuple $\langle l, r, S, D e p\rangle$ where,

- $l$ and $r$ are vertexes in the chart.

- $S$ is a state of an LA.

- Dep is a set of tuples in the form of $\left(D, e_{h}, c_{n}, R\right\rangle$ where $e_{h}$ and $e_{n}$ are edges, $D$ is a quasi-sign(1) and $R$ is a rule schema.

The intuition behind this definition is,

- $S$ plays the role of a non-/terminal in CFG, though it is actually a quasi-sign $(0)$.

- $e_{h}$ and $e_{2}$ denote a head daughter edge and a non-head daugliter edge, respectivoly.

- Dep represents the dependency of an elge and its daughter erlges. Where $\left\langle D, e_{h}, e_{n}, R\right\rangle \in$ Dep, $D$ is a DFS of a transition arc. Basically, Phase 1 parsing creates these tuples, and Phase 2 parsing uses them.

The Phase 1 parsing consists of the following steps. Assume that a word in input has a lexical entry $L_{i}$ and that an $L \Lambda\left\langle Q_{i}, \Lambda_{i}, q_{0}^{i}\right\rangle$ generated from $L_{i}$ is attached to tho word:

1. Create an edge $l_{i}=\left\langle j_{i}, j_{i}+1, q_{0}^{i}, \phi\right\rangle$ in the chart for each $L_{i}$, for appropriate $j_{i}$.

2. For an edge $e_{1}$ whose state is $q_{1}$ in the chart, pick up an edge $e_{2}$ which is adjacent to $e_{1}$ and whose state is $q_{2}$.

3. For a transition arc $\left\langle q_{1}, q, N, D, R\right\rangle$, check if $N$ is unifiable with $q_{2}$.

4. If the unifiability chock is successful, find an edge $d=\left\langle m_{d}, n_{d l}, q, D_{e} p_{d}\right\rangle$ strictly covering $e_{1}$ and $e_{2}$.

5. if there is, replace $d$ with a new edge $\left\langle m_{d}, n_{d}, q, D e p_{d} \cup\left\{\left\langle D, e_{1}, e_{2}, R\right\rangle\right\}\right\rangle$ in the chart.

6. Otherwise,

create a new edge $\left\langle m, n, q,\left\{\left\langle D, e_{1}, e_{2}, R\right\rangle\right\}\right\rangle$ strictly covering $e_{1}$ and $e_{2}$.

7. Go to step 2

\section{Phase 2 Parsing}

The algorithm of Phase 2 parsing is given in Figure 4. The procedure sub-structure is a recursive procedure which takes an edge as input and builds up sub-structures, which is differential foature structures representing modifications to core-structures, in a bottom-11) manner.
The obtained sub-structures are unified with corestructures when 1) the input edge covers a whole input or 2) the edge is a non-hoarl daughter edge of some other edge. Note that the sub-structure treats $s u b(f s(R))$, a feature structure eliminated by the restriction in the generation of $L \Lambda s$, (the (A) part in Figure 4) and frozen goals of DCPs, ly additional evaluation of DCPs. (the (B) part)

Here, we use two terhniques: One is dependency analysis which is embodied by the function $d(p)$ in Figure 4. The other is a partial unification rontine expressed by p.unify in the figure.

The dependency analysis is represented with the function, dep $(F, r s)$, where $f^{\prime}$ is a DFS and $r s$ is a restriction schema used in generation of LAs:

Definition 9 (dep) For a feature structure $F^{\prime \prime}$ and the restriction schema rs, $F=\operatorname{dep}\left(l^{\prime \prime}, r s\right)$ is a maximal feature structure sach that any node $n$ in $F$ satisfies the conjunction of the following lwo conditions:

1. There is a node $n^{\prime}$ in $F^{\prime \prime}$ such that $\operatorname{paths}(n, F)=\operatorname{puths}\left(n^{\prime}, f^{\prime \prime}\right)$ and type $(n)=$ type $\left(n^{\prime}\right)$.

2. Where, A) $n_{d}=n$ or B) $n_{d}$ is a descendant of $n$, paths $\left(n_{d}, F\right)$ contains a path prefixed by one of $\langle$ head-dtr $\rangle$, (non-head-dtr $\rangle$ and 〈goals〉.

3. The disjunction of the following three conditions is satisfied where $A) n_{d}=n$ or $B$ ) $n_{d}$ is a descendant of $n$.

- For some $p \in$ paths $\left(n_{d}, F\right)$, there is a path $p_{r} \in$ rs which prefixes $p$.

- Some $p \in \operatorname{paths}\left(n_{d}, F^{\prime}\right)$ is prefixed by \{goals $\rangle$.

- There is no node $n_{a}$ in $F$ such that i) there is paths $p_{1}, p_{2} \in$ paths $\left(n_{n}, F^{\prime}\right)$ such that $p_{1}$ is prefixed by (syn) or (sem) and $p_{2}$ is prefixed by (head-dtr) or (non-head-dtr $\rangle$, and ii) for any $p \in$ paths $\left(n_{d}, F\right)$ there is $p_{a} \in \operatorname{paths}\left(n_{n}, F\right)$ which prefixes $p$.

Roughly, dep climinates l) the descendant nodes of the node which appears both in syn/sem domains and head-dtr/non-head-dtr domains and 2) the nodes appoaring only in syn/sem domains, except for the node which appears in $s u b(f s(l i))$ or goals domains. In other words, it removes the feature structures that have been already raised to core-structures or other DFSs, except for the structure sharings, and leaves those which will be required by DCPs or $s u b(f s(R))$.

$p_{-} u n i f y\left(F_{1}, F_{2}, r s\right)$ is a partial unification routine where $F_{1}$ and $F_{2}$ are feature structures, and $r s$ is a restriction schema used in generation of LAs. Roughly, it performs unification of $F_{1}$ and $F_{2}$ only for common part of $F_{1}, F_{2}$, and it produces unified results only for the node $n$ in $F_{1}$ if

\footnotetext{
${ }^{5} n_{1}$ is a rescendant of $n_{2}$ in a feature structure $I^{\prime}$ ilf $n_{1} \neq n_{2}$, and there are paths $p_{1} \in$ path $s\left(n_{1}, F^{\prime}\right)$ and $p_{2} \in \operatorname{poth}\left(n_{2}, l^{\prime}\right)$, and $p_{2}$ prefixes $p_{1}$.
} 


$$
\left[\begin{array}{ll}
\text { phon } & \text { "wrote" } \\
\text { syn } & {\left[\begin{array}{ll}
\text { head } & \\
\text { subcat } & \langle N P[1], N P[2]\rangle
\end{array}\right]} \\
\text { sem } & {\left[\begin{array}{ll}
\text { content } & {\left[\begin{array}{ll}
\text { pred } \\
\text { reln } & \text { wrote } \\
\text { agent } & 22 \\
\text { object } & {[1]}
\end{array}\right]}
\end{array}\right]}
\end{array}\right]
$$

Figure 5: A lexical entry for "wrote"

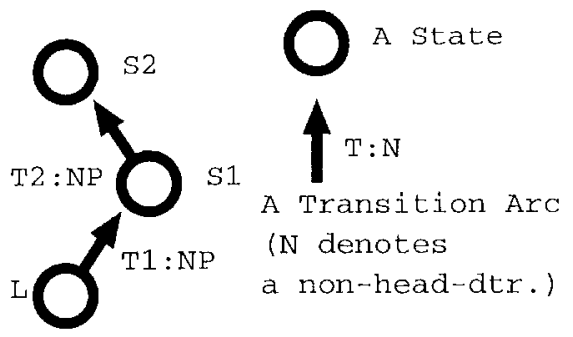

Figure 6: The LA derived from "wrote"

$n$ has a counter part in $F_{2}$. More precisely, it produces the unification results for a node $n$ in $F_{1}$ such that

- there is a path $p \in \operatorname{paths}\left(n, F_{1}\right)$ such that the node reached by $p$ is also defined in $F_{2}$, or

- there is a path $p \in \operatorname{paths}\left(n, F_{1}\right)$ prefixed by some $p_{r} \in$ rs or $\langle$ goals $\rangle$.

Note that a node is unified if its structureshared part has a counter-part in $F_{2}$. Intuitively, the routine produces unified results for the part of $F_{1}$ instantiated by $F_{2}$. The other part, that is not produced by p.unify, is not required at Phase 2 because it is already computed in a state or DFSs in LAs when the LAs are generated. Then, a sign can be obtained by unifying a sub-structure and the corresponding core-structure.

\section{Example}

This section describes the parsing process of the sentence "My colleague wrote a good paper." The LA generated from the lexical entry for "wrote" in Figure 5 is given in Figure 6. The transition arc $T 1$ between the states $L$ and $S 1$ is generated by the rule schema in Figure 1. Note that the query to DCP, freeze $([1]$, append $([1],[2],[3]))$, is used to obtain union of indices values of daughters and the result is written to the indices values of the mother sign. During the generation of the transition arc, since the first argument of the query is [ $\perp$ ], it is frozen. The core-structures and the dependencyanalyzed DFSs that augment the LA are shown in Figure 7 . Wo assume that we do not use any restriction, i.e., for any lexical entry $l$ and rule schemata $R, \operatorname{sub}(l)=[\perp]$ and $\operatorname{sub}(f s(R))=[\perp]$.

Note that, in the DFSs, the already raised feature structures are eliminated and, that the DFS of the transition arc $T$ contains the frozen query as the goals.

Assume that the noun phrases "My colleague" and "a good paper" are already recognized by a parser. At phase 1, they are checked if they are unifiable to the condition of transition arcs $T 1$ and $T 2$, i.e., the $N P$ s which are non-head daughters
$S 2$

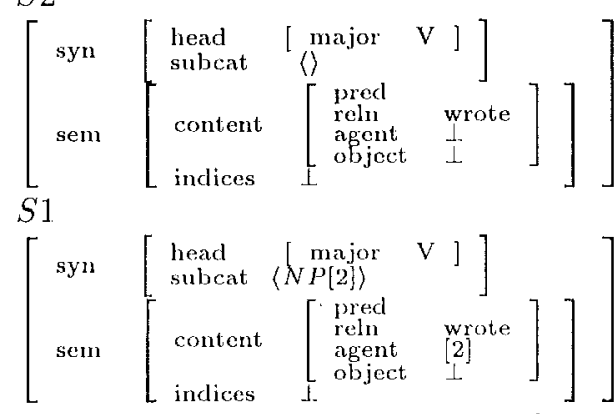

The dependency-analyzed $D F S$ of $T 2$

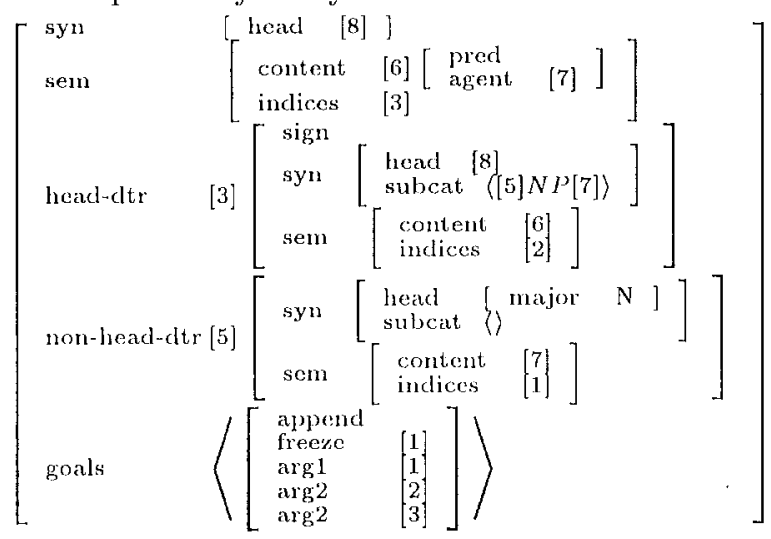

The dependency-analyzed $D F S$ of $T 1$

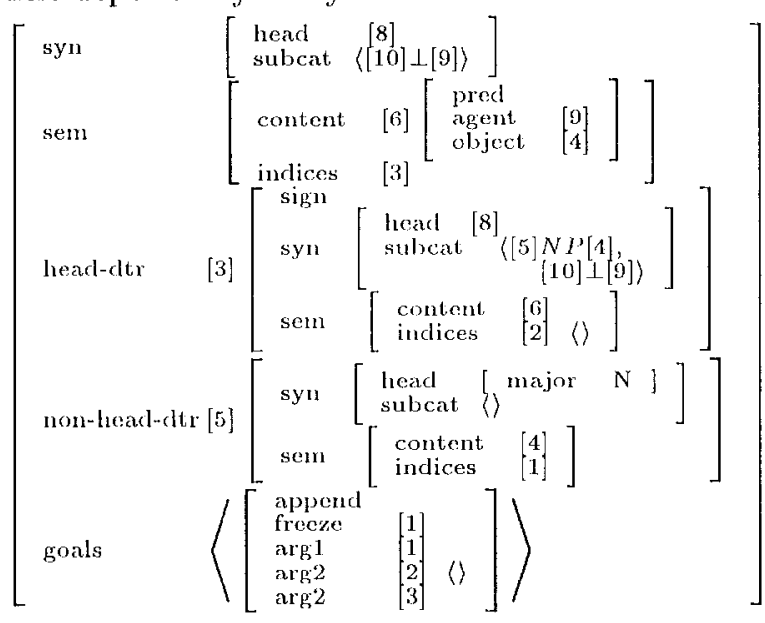

Figure 7: States and DFSs in the LA in Figure 6

The sub-structure for $S 2$

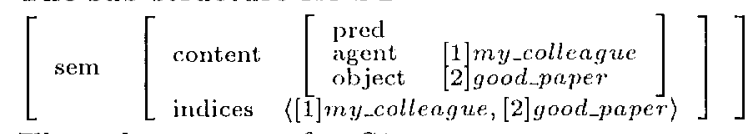

The sub-structure for $S 1$

$\left[\operatorname{sem}\left[\begin{array}{ccc}\text { content } & {\left[\begin{array}{c}\text { pred } \\ \text { object }\end{array}[1] \text { good_paper }\right.}\end{array}\right]\right]$

The goals, head-dir, non-head- dir values are omitted.

Figure 8: The sub-structures obtained in the parsing 


\begin{tabular}{|c|c|c|c|}
\hline Parsing Algorithm & $\begin{array}{l}\text { Type of sentences } \\
\text { (\# of sentences) }\end{array}$ & Avg Length (word) & Avg I'ime (sec) \\
\hline Phase 1 only & all $(70)$ & 19.2 & $1.25(1.12)$ \\
\hline Phase 1 \& Phase 2 & all $(70)$ & 19.2 & $3.00(1.65)$ \\
\hline Phase 1 \& Phase 2 & only successful (43) & 18.8 & $3.37(1.84)$ \\
\hline $\begin{array}{l}\text { Phase } 1 \text { \& } \\
\text { naive application of rule schemata }\end{array}$ & only successful & 17.13 & $55.09(9.27)$ \\
\hline $\begin{array}{l}\text { Phase } 1 \text { \& } \\
\text { naive application of rule schematia }\end{array}$ & (5) & 31.4 & $1093.22(82.12)$ \\
\hline
\end{tabular}

$\Lambda$ bracketed time inclicates non-GC execution tine. ']'he experiments was performed on Sparcitation 20 with $128 \mathrm{ML}$ RAM.

Figure 9: Experiments on a Japanese newspaper( Asahi Shinbuu)

of $\$ 1$ and $S 2$. Since all the nuifiability chockings are successful, Phaso 1 parsing produces the parse tree whose form is presented in Figure 3 . The Phase 2 parsing produces the sub-structures in Figure 8. Note that the frozen goals are evaluated and the indices values have appropriate values. $A$ parsing result is obtained by unifying the sub-structure for $S 2$ with the corresponding core-structure.

The amount of the feature structure nodes generated during parsing are reduced compared to the case of the naive application of rule schemata presented in section 2. The important point is that they contain only either the part in the DFSs that was instantiated by head dangliters' sub-structures, and non-head claughters' corestructures and sub-structures, or the part that contributes to the DCl's evaluation. The foature structure that does not appear in a sub-structure appears in the corresponding core-structure. Sec Figure 7. Because of these properties, the correctness of our parsing metlood is guaranteed. (Torisawa and Tsujii, 1996).

\section{Experiments}

We have implemented onr parsing method in Common Iisp Object System. Improvement by our method has been measured on 70 randomly selected Japaneso sentences from a nowspapor (Asahi Shinbun). The used grammar consists of just 5 rule schemata, which are generated from principles and rewriting rules, and 55 default lexical entries given for each part of speech, with 44 manually tailored lexical entries. The total num ber of states in the $L \Lambda$ s compiled from them was 1490. The grammar loes not have a semantic part. The results are prosented in Figure 9. Our grammar produced possible parso trees for $43 \mathrm{sen}-$ tences $(61.4 \%)$. We compared the execution time of our parsing methor and a more naive algorithm, which performs l'hase 1 parsing with LAs and applys rule schemata to completed parse trees in the naive way described in Section 2. As the naive algorithm caused thrashing for storage in $\mathrm{GC}$, it is pointless to compare those figures simply. However, it is obvious that our method is much faster than the naive one. We could not measure the execution time for a totally naive algorithm which builds parse trees without LAs because of thrashing.

\section{Conclusion}

We have presented a two-phased parsing method for HIPSG. In the first phase, our parser produces parse trees using lexical Fintry Automata compiled from lexical cutrics. In the second phase, only the feature structures which must be computed dynamically are computed. As a result, anount of the frature structures unified at parsing-time is reduced. We also showed the effect of our optimization techniques by a serios of experiments on a roal work text.

It can be noticed that each transition arc of the compiled $\mathrm{I} A \mathrm{~s}$ can be seen as a rowriting rule in CIFG (or a dotted notation in a chart parser.) We believe this can open the way to integrate several methods developed for $\mathrm{CH} G$, including the insideontsicle algorithm for grammas learning or disanbiguation, into an HPSG framowork. We also believe that, by pursuing this direction for optiniming HPSG parsers, we can reach the point where grammar learning from corpora can be done with concise and linguistically well-defined core granmar.

\section{References}

Bob Carpenter. 1992. The Logic: of Typed Feature Structures. Cambridgo University Press.

Keiko Horiguchi, Kentaro Torisawa, and Jun'ichi Tsujii. 1995. Automatic accuisition of content words using an HPSG-based parser. In $N L$ PRS'95.

Robert Kasper, Bernd Kiefor, Klaus Netter, and K. Vijay-Shanker. 1995. Compilation of IMPSG to TAG. In ACL 95.

Carl Pollard and Ivan A. Sag. 1987. InformationBased Syntax and Semantics Vol.1. CSLI lecture notes no. 13.

Carl Pollard and Ivan A. Sag. 1993. MeadDriven Phrase Structure Grammar: University of Chicago Press and CSLI Publications.

Stuart, C. Shicber. 1985. Using restriction to extend parsing algorithms for complex feature based formalisms. In ACL85.

Kentaro Torisawa and Jun'ichi 'I'sujii. 1996. Offline raising, dependency analysis and partial unification. In 7hird International Conference on HPSG. In the procedings of TALN' 96 . 